Ferree BA, Stern PJ, Jolson RS, et al. Deep venous thrombosis after spinal surgery. Spine 1993; 18:315-9.

Ferree BA, Wright AM. Deep venous thrombosis following posterior lumbarspinal surgery. Spine 1993; 18:1079-82.

McNally MA, Mollan RAB. Venous thromboembolism and orthopaedic surgery. J Bone Joint Surg [Br] 1993; 75-B:517-9.

THRIFT (Thromboembolic Risk Factors Consensus Group). Risk of and prophylaxis for venous thromboembolism in hospital patients. $\mathrm{Br}$ Med J 1992; 305:567-74.

Authors' reply:

Sir,

Thank you for the opportunity to reply to Mr Dove. He has raised an important issue affecting many orthopaedic surgeons. Some consensus committees regard orthopaedic surgery as a homogenous specialty with a uniformly high risk of thromboembolism. It is clear that several subspecialties, such as hand surgery and paediatric orthopaedics, have a very low incidence of deep-vein thrombosis and do not require the use of prophylaxis. We could not cover specific subgroups in detail, but we agree that spinal surgery should not be included in prophylactic policies designed for total joint replacement and fractures.

The consequences of anticoagulant-induced bleeding are considerably greater in spinal surgery. Anticoagulants should only be used in selected cases with a particularly high risk of deep-vein thrombosis, such as after previous thromboembolism. We use intraoperative intravenous Dextran 70 for spinal cases rather than heparin or low-molecular-weight heparin.

Mr Dove has quoted two significant papers which advocate mechanical prophylaxis in spinal surgery. Enhancement of venous blood flow by physical means is a safe and effective option. We support the wider use of physical methods of prevention in all areas of orthopaedic surgery, especially for patients with a high risk from bleeding complications.

M. A. MCNALLY, FRCS

R. A. B. MOLLAN, MD, FRCS, FRCS I

Musgrave Park Hospital

Belfast, UK.

\section{MANAGEMENT AFTER ARTHROSCOPY}

Sir,

We were pleased that Birch et al in their recent article in the July 1993 issue entitled 'Anti-inflammatory drug therapy after arthroscopy of the knee' (1993; 75-B:650-2) included assessment of the efficacy of physiotherapy after arthroscopy, but were concerned about the design of their trial. To reflect current practice in this country, the NSAID group was given a specified seven-day course of drugs. The physiotherapy group had daily treatment continued to full functional recovery, which is unlikely to be standard in this country.

The frequency and content of physiotherapy should always be directed by the pathology: it should be very different after arthroscopy in patients with degenerative changes than in those with recent ACL rupture. Birch et al did not report the mode of treatment. Were the patients given electrotherapy, a defined, supervised exercise regime, reinforcement of home exercises or just counselled? They report the results of a non-existent, 'generic physiotherapy'. Their conclusion that such physiotherapy appears to be ineffective after arthroscopy, is meaningless.

D. BEARD, MSc, MCSP

Nuffield Department of Orthopaedic Surgery

J. MOSER, MSc, MCSP

C. CRAWFORD, MCSP

Physiotherapy Department

Nuffield Orthopaedic Centre NHS Trust

Oxford, UK.
Birch NC, Sly C, Brooks S, Powles DP. Anti-inflammatory drug therapy after arthroscopy of the knee: a prospective, randomised, controlled trial of diclofenac or physiotherapy. J Bone Joint Surg [Br] 1993; 75 B:650-2.

Author's reply:

Sir,

Thank you for allowing us to reply to the letter from Beard and his colleagues. We regret that there was an error in our paper: outpatient physiotherapy was not daily, but varied according to the clinical condition of the patient. On average, patients in the physiotherapy group had 3.5 treatments ( 1 to 11$)$, ranging from a single visit to repeat attendances over several weeks. We apologise for this error.

We were trying to assess benefit from the routine referral of patients for physiotherapy after arthroscopy. The actual treatments prescribed by our physiotherapists were dictated by the clinical condition of the patient; they included exercises and electrotherapy. We agree with Beard et al that physiotherapists are at liberty to prescribe whatever treatments they deem necessary for individual patients; we did not consider that this point needed specific mention as all physiotherapists and most surgeons are aware of it.

We were comparing two methods of management and did not therefore distinguish between different treatments, accepting that our physiotherapists would use the most appropriate methods for each case. This is not 'generic physiotherapy'.

Directed treatment is logical for both groups: a few of our patients with synovitis after a meniscal injury improved more quickly in the first two weeks with non-steroidals and some patients with poor quadriceps function or a poor range of motion benefited more from physiotherapy. The numbers were too small for statistical analysis but it seems probable that treatment should be carefully selected. This was intended to be the essential message of our study.

\section{N. C. BIRCH, FRCS}

Potters Bar

Hertfordshire, UK.

\section{SPINAL STENOSIS}

Sir,

I commend Sanderson and Wood (1993; 75-B:393-7) for investigating the efficacy of surgery for lumbar spinal stenosis in elderly patients, and I believe that they are correct to conclude that "the results of local decompression for sciatica and neurogenic claudication in the elderly are good"'.

I would, however, like to have seen more use of statistics. The data were presented as numbers and percentages only - it would have been better to have given $p$ values to account for the role of chance in the results. The only consideration of confounding variables was to give results based on diagnosis and then to list the number and percentage in each category. Their outcome measure was an ordinal scale and it would have been more effective to have used Mantel-Haenszel chi-squared testing to measure both the level of significance and to control for confounding.

The authors should have given more details of the different categories of outcome. They listed the variables, including pain, patient satisfaction, analgesic use, and walking distance but they failed to state how they defined excellent, good, or fair outcomes. These designations thus appear to be highly subjective and could lead to a reliability bias. Their findings require additional statistical tests before recommendations can be made.

\section{SCHWARTZ}

Meharry Medical College

Nashville

Tennessee, USA 
Sanderson PL, Wood PLR. Surgery for lumbar spinal stenosis in old people. J Bone Joint Surg [Br] 1993; 75-B:393-7.

\section{Authors' reply:}

Sir,

The main purpose of our paper was to show that the often vague symptoms of spinal stenosis in the elderly should not be dismissed, because surgery can help. This group of patients is often ignored because the value of surgery is not appreciated. We accept that a chi-squared test could have been used to measure the significance of the various factors, but our criteria for the results were stated in the paper.

We used a simple outcome measure:

excellent - no leg pain;

good - less leg pain than before surgery; and

poor - no change or worse leg pain.

We also reported other data such as back pain, walking distance and the use of analgesia, as recommended by Turner et al (1992). This makes it possible for the reader to do his own statistical tests. We felt that the results which we gave justified our conclusion; 27 of the 31 patients were satisfied and 25 were improved on the criteria which we used.

\section{P. L. SANDERSON, FRCS \\ P. L. R. WOOD, FRCS \\ Royal Hallamshire Hospita \\ Sheffield, UK}

Turner JA, Ersek M, Herron L, Deyo R. Surgery for lumbar spina stenosis: attempted meta-analysis of the literature. Spine 1992; 17:1-8.

\section{PAIN RELIEF AFTER DISCECTOMY}

Sir,

We read with interest the paper by Milligan et al in your September 1993 issue (1993; 75-B:769-71) showing that the use of bupivacaine significantly reduces morphine consumption after discectomy. They state in their discussion that hourly morphine consumption in the study group remained below that of the control group for the duration of the study. Inspection of the data presented in the paper does not support this.

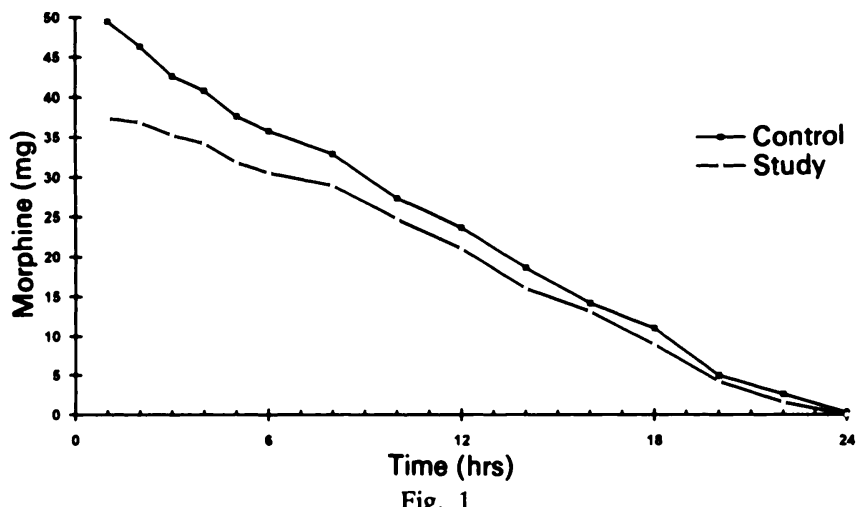

We have calculated the hourly consumption of morphine $o_{\text {i }}$ : the basis of the difference in the mean cumulative consumption: after eight hours there is no obvious difference between the two groups. This is clearly seen when their Figure 1 is reversed, plotting the amount of morphine remaining to be used against time (Fig. 1). We cannot assess statistical significance, but suggest that even a statistically significant reduction of under $3 \mathrm{mg}$ in the total consumption over the last 12 hours is of little clinical relevance.
We also have reservations on the extent of the 'blinding'. The nursing staff who provided the formal assessment of pain control were blinded, but the surgeon and anaesthetist, who presumably continued their care of the patient into the postoperative period, were not. Pain assessment, even using visual analogue scoring, is notoriously subjective and patients who have consented to take part in such studies are often very receptive to clues as to which treatment they have received, and may unwittingly modify their responses.

Almost all of the excess morphine requirement of the control group occurred in the first six to eight hours, according well with the known plasma half-life of bupivacaine of just under three hours (Calvey and Williams 1991). In the absence of a blinded control infiltration, any subsequent morphine sparing could result from lack of blinding, the residual effect of the bupivacaine or the physical effect of tissue infiltration, rather than the suggested reduction of spinal cord hyperexcitability.

\section{J. E. RISDALL, FFARCS I}

C. G. JOHNSTON, FFARCS I

RNH Haslar

Gosport, UK.

Calvey TN, Williams NE. Principles and practice of pharmacology for anaesthetists. 2nd ed. Oxford. Blackwell Scientific Publications, 1991:241.

Milligan KR, Macafee AL, Fogarty DJ, et al. Intraoperative bupivacaine diminishes pain after lumbar discectomy: a randomised double-blind study. J Bone Joint Surg [Br] 1993; 75-B:769-71.

Authors' reply:

Sir,

We agree with Risdall and Johnston that wound infiltration with bupivacaine produced most of its benefits in the first 12 hours. This is to be expected since the passage of time will make it increasingly difficult to show a difference in postoperative pain relief.

We were particularly concerned about a possible 'rebound effect' causing increased morphine consumption when the bupivacaine ceased to be effective, one criticism of previous studies of wound infiltration (McQuay 1992). This rebound did not occur, the bupivacaine group reporting less pain despite their lower morphine consumption.

We agree that it might have been better to inject saline, for example, into the wound of the control group. Because neither the patients nor those carrying out the postoperative assessments knew what had been given, we do not consider that this invalidates our main conclusion: intraoperative wound infiltration with bupivacaine does provide significant improvement in postoperative pain relief.

K. R. MILLIGAN, MD, FFARCS

A. L. MACAFEE, MD, FRCS

R. G. H. WALLACE, MCh, MD, FRCS

Musgrave Park Hospital

Belfast, UK.

McQuay HJ. Editorial: pre-emptive analgesia. Br J Anaes 1992; 69:1-3.

\section{HYPOTENSION DURING CEMENTED ARTHROPLASTY}

Sir,

We read with interest the article by Wheelwright et al in your September 1993 issue entitled 'Hypotension during cemented arthroplasty' (1993; 75-B:715-23). We agree with their recommen- 